\title{
Plasmon resonances of aluminum nanoparticles and nanorods
}

\author{
Y. Ekinci, ${ }^{1, a)}$ H. H. Solak, ${ }^{2}$ and J. F. Löffler ${ }^{1}$ \\ ${ }^{1}$ Laboratory of Metal Physics and Technology, Department of Materials, ETH Zurich, \\ Wolfgang-Pauli-Str. 10, 8093 Zurich, Switzerland \\ ${ }^{2}$ Laboratory for Micro- and Nanotechnology, Paul Scherrer Institute, 5232 Villigen-PSI, Switzerland
}

(Received 24 June 2008; accepted 20 August 2008; published online 23 October 2008)

\begin{abstract}
We report experimental and theoretical analysis of the plasmonic resonances of Al nanoparticles and nanorods. Ordered nanoparticle arrays with well-defined shapes and narrow size distributions are fabricated on quartz substrates over large areas using extreme ultraviolet interference lithography. The structures, which have sizes down to $40 \mathrm{~nm}$, exhibit strong and sharp particle plasmon resonances in the near and deep-UV ranges. A comprehensive theoretical analysis carried out using dipolar approximation and finite-difference time-domain methods shows good overall agreement with measurements while revealing the dependence of the optical response of Al structures on the fabrication conditions. The results demonstrate the suitability of using $\mathrm{Al}$ as a plasmonic material in the UV range and the feasibility of extending applications of plasmonics, such as surface-enhanced Raman spectroscopy, down to the deep-UV range. (C) 2008 American Institute of Physics.
\end{abstract}

[DOI: $10.1063 / 1.2999370]$

\section{INTRODUCTION}

Metallic nanoparticles scatter and absorb light strongly at certain wavelengths due to the resonant excitation of charge density oscillations known as localized particle/ surface plasmons. ${ }^{1,2}$ Potential applications of these plasmon resonances, such as in Raman and fluorescence spectroscopy, biochemical sensors, and nanophotonics, have led to an increasing interest in nanoparticles and their plasmonic properties. ${ }^{3-5}$ Although plasmonic properties of many metallic nanoparticles, in particular $\mathrm{Au}$ and $\mathrm{Ag}$, have been investigated extensively, the literature on $\mathrm{Al}$ nanoparticles is surprisingly limited. Whereas $\mathrm{Au}$ and $\mathrm{Ag}$ exhibit interband absorptions below the wavelengths of about 590 and 350 $\mathrm{nm},{ }^{6}$ respectively, $\mathrm{Al}$ has low absorption down to a wavelength of $200 \mathrm{~nm}$ due to its free-electron-like character and high bulk-plasmon frequency. ${ }^{7}$ The accessibility of the spectrum in the 200-500 $\mathrm{nm}$ range makes $\mathrm{Al}$ an excellent and perhaps the only choice for applications such as broad-band wire-grid polarizers. ${ }^{8,9}$ Being considered as good freeelectron metal, calculations on the plasmonic resonances of $\mathrm{Al}$ can be found in textbooks. ${ }^{1,2}$ For a comparison of $\mathrm{Al}$ to common plasmonic materials, the extinction spectra of spherical $\mathrm{Au}, \mathrm{Ag}$, and $\mathrm{Al}$ nanoparticles with diameters of 40 $\mathrm{nm}$ in a silica matrix are shown in Fig. 1(a). The spectra were calculated using Mie's theory, which provides exact analytical solutions for spherical particles. ${ }^{1,2}$ The extinction peaks in the blue and green regions result from the resonant excitation of the dipolar plasmon modes of $\mathrm{Ag}$ and $\mathrm{Au}$ nanoparticles. An $\mathrm{Al}$ nanoparticle of the same size exhibits a peak at about $250 \mathrm{~nm}$ due to the dipolar plasmon resonance and another peak at about $190 \mathrm{~nm}$ resulting from the quadrupolar mode. In Fig. 1(b), the extinction spectra of $\mathrm{Al}$ nanospheres with different diameters are presented. With increasing par-

\footnotetext{
a) Author to whom correspondence should be addressed. Electronic mail: yasin.ekinci@mat.ethz.ch.
}

ticle size, the Al spectrum exhibits a strong redshift and broadening due to retardation effects and emergence of higher order plasmon modes. Compared with $\mathrm{Au}$ and $\mathrm{Ag}$ nanoparticles of similar dimensions, the redshift of the plasmon peaks and observation of high-order plasmon modes are relatively significant for $\mathrm{Al}$ nanoparticles. For instance, for $\mathrm{Au}$ nanoparticles, the redshift of the dipolar resonance is only $150 \mathrm{~nm}$ when the particle diameter is increased from 20 to $140 \mathrm{~nm}$ as shown in Fig. 1(c).

Experimental studies on the plasmonic properties of $\mathrm{Al}$ face certain difficulties. Whereas chemical synthesis methods for $\mathrm{Au}$ and $\mathrm{Ag}$ have reached an advanced stage, providing particles of different sizes and shapes, the synthesis of $\mathrm{Al}$ nanoparticles with satisfactory size dispersion and without significant agglomeration remains a challenge. Pronounced plasmon peaks of $\mathrm{Ag}$ and $\mathrm{Au}$ can be obtained rather easily by roughening their surfaces or through island formation in thin films. Al particle plasmon resonance, however, has a stronger dependence on particle size and shape, as seen above, with strong redshift and emergence of broad features as the particle size grows. In random nanoparticle ensembles, inhomogeneous broadening as a consequence of shape and size variations smears out all the peaks. This is the reason for lack of any resonant features, unlike for $\mathrm{Au}$ and $\mathrm{Ag}$ thin films, for deposited Al thin films with significant island formation as reported even in recent studies. ${ }^{10}$ In the small-particle size limit $(d<10 \mathrm{~nm})$, only the dipolar plasmon mode, which is rather insensitive to the size distribution, remains. However, the resonance peak is expected to occur at about $180 \mathrm{~nm}$ (in a silica matrix). Thus, for an explicit observation of the plasmon peak, optical experiments in the vacuum ultraviolet region are required. The recent availability of nanofabrication tools such as e-beam and focused ion beam lithography has enabled extensive experimental studies on the properties of nanoparticles. In general, ensembles of particle arrays have been fabricated and characterized with a microscope coupled to a spectrometer. However, these tools do not typically 


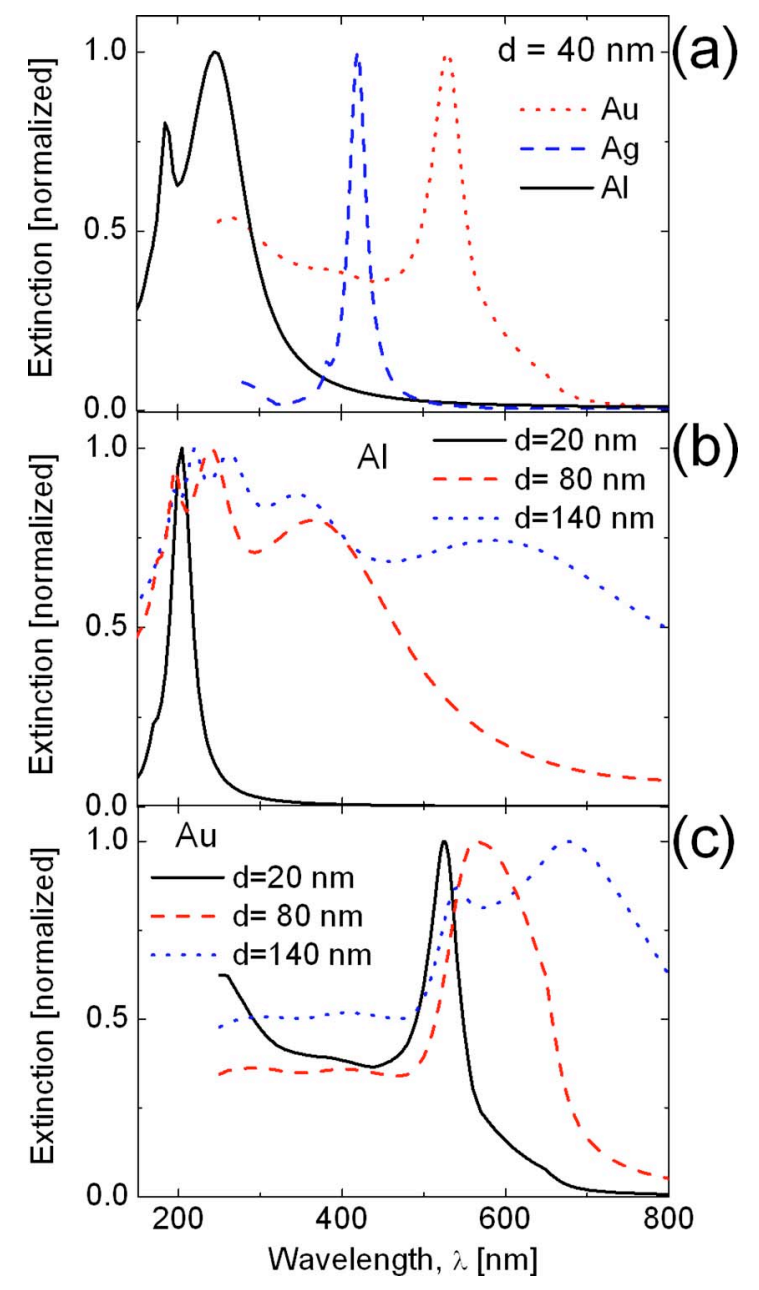

FIG. 1. (Color online) (a) Calculated extinction spectra of spherical Au, Ag, and $\mathrm{Al}$ nanoparticles with a diameter of $40 \mathrm{~nm}$ in a silica matrix. (b) Calculated extinction spectra of spherical Al nanoparticles with diameters of 20 , 80 , and $140 \mathrm{~nm}$ in a silica matrix. (c) Calculated extinction spectra of spherical Au nanoparticles with diameters of 20,80 , and $140 \mathrm{~nm}$ in a silica matrix. Calculations are based on Mie's formulae; for $\mathrm{Au}, \mathrm{Ag}$, and Al the optical constants provided in Refs. 6 and 7 were used.

cover the UV range needed for the measurements on $\mathrm{Al}$ nanoparticles. This problem can be circumvented by fabricating ensembles of nanoparticles over large areas, and thus the use of a UV-compatible microscope can be avoided. Nevertheless, this fabrication method is rather time consuming because of its serial writing nature.

Significant interest has been paid to the optical properties of Al nanoparticles in the far-infrared region. Al particles smaller than $10 \mathrm{~nm}$ exhibit anomalous far-infrared absorption, ${ }^{11,12}$ which has been attributed to quantum size effects $^{13}$ and the effects of oxide overlayer. ${ }^{14}$ Another interesting effect that has attracted attention is the diminishing of the interband absorption at $850 \mathrm{~nm}$ wavelength in small $\mathrm{Al}$ clusters, ${ }^{15-17}$ resulting from the breakdown of the band structure. Broad absorption peaks attributed to surface plasmon modes were measured for nanoporous thin films, ${ }^{18}$ for thermally evaporated discontinuous films, ${ }^{19,17}$ and for hollow nanocaps. ${ }^{20,21}$ Surface plasmon modes were also observed in electron energy loss spectroscopy experiments of vapordeposited Al nanoparticles. ${ }^{22,23}$ Recently, enhanced transmis- sion through $\mathrm{Al}$ hole arrays has been observed and attributed to the resonant excitation of localized and extended surface plasmons. $^{24}$

In this paper we report our experimental observations on the plasmonic behavior of $\mathrm{Al}$ nanoparticles and nanorods. Nanostructures over large areas with well-defined shapes and narrow size distributions have been obtained using extreme ultraviolet (EUV) interference lithography. Fabrication of $\mathrm{Al}$ nanostructures with narrow size distribution is essential for obtaining sharp plasmon resonances because of the aforementioned significance of inhomogeneous broadening for $\mathrm{Al}$ nanoparticles. Moreover, this fabrication method provides large-area samples that simplify spectroscopic measurements in the UV region. The fabricated particles exhibit sharp particle plasmon resonances down to the deep-UV region. We compare experimental results with theoretical predictions obtained using dipolar approximation and finite-difference time-domain (FDTD) calculations and discuss the validity of the dipolar approximation. The effects of the natural oxide layer and the optical constants are investigated using these theoretical models.

\section{EXPERIMENTAL DETAILS}

To obtain Al nanoparticles we used EUV interference lithography, ${ }^{25}$ physical vapor deposition, and lift-off techniques. Two-dimensional arrays of $\mathrm{Al}$ nanoparticles with different sizes, shapes, and periods were fabricated on quartz substrates. Sample preparation started with the spin coating of 80-nm-thick polymethylmethacrylate (PMMA) on silica (HPFS) wafers and with baking at $180{ }^{\circ} \mathrm{C}$ for $3 \mathrm{~min}$. Subsequently, hydrogen silsesquioxane (HSQ) of $30 \mathrm{~nm}$ in thickness was spin coated on the PMMA film and the samples were baked at $90{ }^{\circ} \mathrm{C}$ for $3 \mathrm{~min}$. The EUV exposures were carried out at the XIL beamline of the Swiss Light Source (SLS) at the Paul Scherrer Institute, Switzerland. The interference setup uses transmission gratings and coherent EUV light of $13.4 \mathrm{~nm}$ wavelength generated by an undulator source. ${ }^{25}$ Following the EUV exposure, HSQ photoresist ${ }^{26}$ was developed in a tetramethyl ammonium hydroxide (TMAH) solution with a normality of 2.6 , resulting in hole patterns in HSQ. Reactive ion etching was used to transfer the pattern into the PMMA layer. This step produces a bilayer structure with an undercut that facilitates the subsequent lift-off process. Thermal evaporation of aluminum was performed at a base pressure of better than $1 \times 10^{-6} \mathrm{mbar}$ with a deposition rate of about $0.5 \mathrm{~nm} / \mathrm{s}$. Finally, the lift-off process was carried out in an acetone bath with ultrasonic agitation. Figure 2 shows scanning electron microscopy (SEM) images of typical samples. For all samples the metal thickness was kept constant at $30 \mathrm{~nm}$. The size of the smallest fabricated particles was $40 \mathrm{~nm}$. As seen in the figure, the particles possess some roughness due to the finite grain size of the $\mathrm{Al}$ crystals making up the nanoparticles. The area of the particle arrays varied in the $0.25-1 \mathrm{~mm}^{2}$ range, which was large enough for characterization without any focusing optics. Extinction spectra (extinction $=1$-transmission) of the nanoparticle arrays were measured using a standard UV/ visible/near infrared (NIR) spectrometer in the transmission 

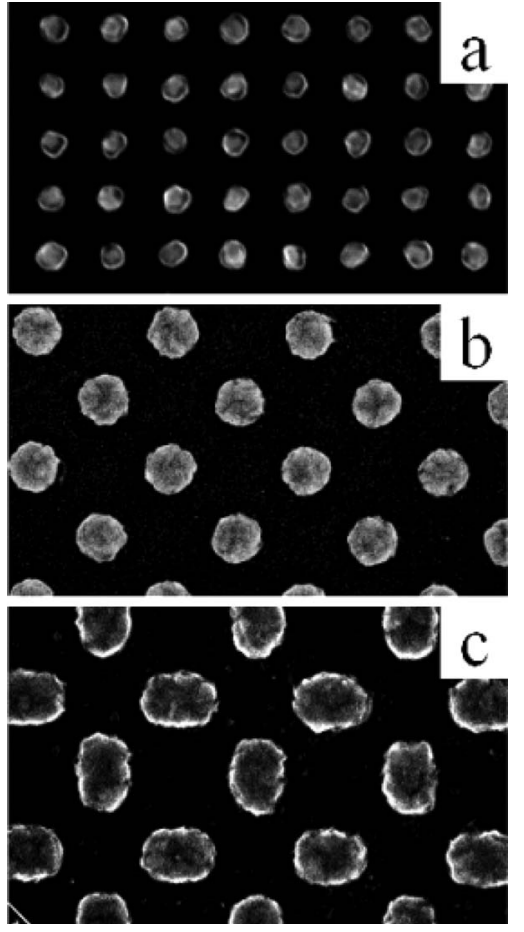

FIG. 2. SEM images of Al nanoparticle arrays (a) with an average particle diameter of $40 \mathrm{~nm}$ and unit cell dimensions of 90 and $100 \mathrm{~nm}$, respectively; (b) with an average diameter of $138 \mathrm{~nm}$ and a period of $280 \mathrm{~nm}$; and (c) with ellipsoidal particles with principle axes of $130 \times 94 \mathrm{~nm}$ and a period of $200 \mathrm{~nm}$. The particle thickness for all samples is $30 \mathrm{~nm}$.

mode with a spectral resolution of $2 \mathrm{~nm}$ at normal incidence and with unpolarized light. The diameter of the light spot was always kept smaller than the sample size.

\section{RESULTS AND DISCUSSIONS}

The measured extinction spectrum of an $\mathrm{Al}$ nanoparticle array with particle diameter of $40 \mathrm{~nm}$ and lattice constants of 90 and $100 \mathrm{~nm}$ in the two orthogonal directions [Fig. 2(a)] is plotted in Fig. 3. The spectrum exhibits a sharp and narrow peak at about $270 \mathrm{~nm}$. This resonant peak can readily be attributed to the dipolar mode of the surface plasmon resonance of the nanoparticles.

We calculated the extinction spectra of the structure using the FDTD method. ${ }^{27}$ FDTD calculations were made us-

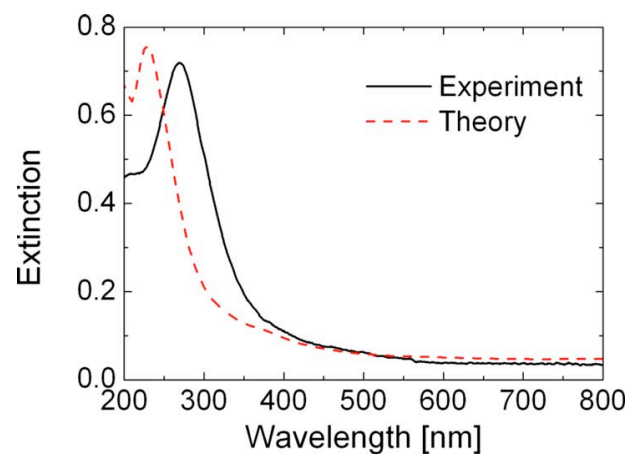

FIG. 3. (Color online) Experimental and theoretical extinction spectra of the Al nanoparticle array with a diameter of $40 \mathrm{~nm}$, a thickness of $30 \mathrm{~nm}$, and lattice constants of 90 and $100 \mathrm{~nm}$. For FDTD calculations, the optical constants tabulated in Ref. 7 were used. ing a code that employs monochromatic plane-wave excitation. Along the axes parallel to the surface plane, periodic boundary conditions were set at the boundaries of the simulation domain, which is equivalent to a single unit cell of the periodic particle array. Absorbing boundary conditions at both ends of the cell along the propagation axis were realized by using perfectly matched layers. ${ }^{27}$ The grid size of the simulation domain was $1 \mathrm{~nm}$. The $\mathrm{Al}$ nanoparticles were modeled as cylinders of $40 \mathrm{~nm}$ in diameter and $30 \mathrm{~nm}$ in height on a semi-infinite quartz substrate. Tabulated values of complex dielectric constants of Al given in Ref. 7 were used in the calculation. A comparison of the experimental and theoretical spectra plotted in Fig. 3 reveals a good agreement in general, notwithstanding a small discrepancy of $40 \mathrm{~nm}$ in the peak position.

The difference may be due to material properties and/or geometrical factors. The dielectric constants used in the FDTD calculations occasionally differ somewhat from the actual dielectric constants of the sample. Moreover, in the FDTD model, the nanoparticles are assumed to be identical and perfect cylinders, whereas the fabricated ones have some shape variations due to the finite grain size of evaporated aluminum. In addition, there is about 5\% size dispersion over the entire area of the particle array. The finite size and shape dispersion lead to inhomogeneous broadening in which the experimental peak width is slightly broader than the theoretical one, as seen in Fig. 3. Moreover, in the FDTD model, the natural oxide layer of $\mathrm{Al}$ is not taken into account. From optical measurements on $\mathrm{Al}$ surfaces ${ }^{28}$ and transmission electron microscopy (TEM) analysis of Al nanoparticles, ${ }^{29}$ it is well known that a thin oxide layer of about $2-3 \mathrm{~nm}$ thickness forms on the metal surface upon exposure to air. Modeling a thin oxide layer in FDTD calculations would have required subnanometer grid sizes and thereby impractically long computing times.

To investigate the potential role of the aforementioned factors to the observed discrepancy between experiment and theory, we performed further calculations using different approaches. In Fig. 4, extinction spectra calculated with various theoretical models are shown, and the FDTD calculations are also plotted for comparison. Note that the spectral range is 160-500 nm in this figure and FDTD calculations show a new peak that emerges from quadrupolar resonance with a shorter resonance wavelength than the dipolar resonance. Dipolar approximation in the quasistatic limit is a commonly used method to explain the optical properties of metallic particles with sizes much smaller than the wavelength. ${ }^{1,2}$ Analytical formulae are available for spherical and elliptical particles as well as core/shell structures. ${ }^{1,2}$

The calculated extinction spectrum of $\mathrm{Al}$ nanoparticles with an oblate shape with principle axes of 40 and $30 \mathrm{~nm}$ using dipolar approximation is shown in Fig. 4. The spectrum shows a single peak at about $210 \mathrm{~nm}$ wavelength while the quadrupolar resonance is missing because this method takes only the dipolar mode into account. In this model, nanoparticles are single and isolated particles, and therefore the coupling between the nanoparticles is neglected. Dipolar coupling between the particles is expected to cause small peak shifts at present particle separation distances. ${ }^{30,31}$ More- 


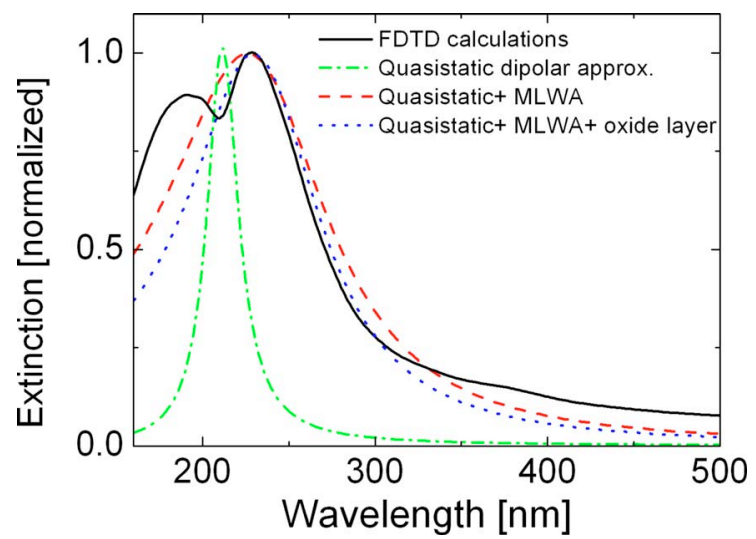

FIG. 4. (Color online) Comparison of the theoretical extinction spectra of Al nanoparticles with an oblate shape with principle axes of 40 and $30 \mathrm{~nm}$ calculated using different models. The solid line (black) denotes FDTD calculations; the dash-dotted (green) line denotes the calculations based on dipolar approximation; the dashed line (red) denotes dipolar approximation calculations with MLWA correction; the dotted line (blue) denotes dipolar approximation with MLWA correction and assuming that the nanoparticle of same total size is coated with 3 -nm-thick $\mathrm{Al}_{2} \mathrm{O}_{3}$. The optical constants provided in Ref. 7 were used.

over, the inhomogeneous dielectric environment of the nanoparticle cannot fully be taken into account. Therefore, the weighted average of the dielectric constants of the air and the substrate is taken as the dielectric constant of the immediate environment of the nanoparticle. This approximation is found to be successful for describing the experimental data of nanoparticles on substrates. ${ }^{31}$ The quasistatic dipolar model results in a narrower and blueshifted peak in comparison to the FDTD model (see dashed-dotted line in Fig. 4). Since this quasistatic approximation ignores dynamic effects, it holds only for small nanoparticles. Although this approximation is widely used to describe the plasmonic resonances of $\mathrm{Au}$ and $\mathrm{Ag}$ nanoparticles of similar size, size-dependent factors such as retardation effects are relatively significant for Al nanoparticles, as demonstrated in Fig. 1(b). An accurate approximation to correct the particle size and radiation damping effects is the so-called modified-long-wavelength approximation (MLWA), ${ }^{31,32}$ which provides simple expressions for extinction cross sections. The quasistatic result obtained in Fig. 4 is corrected with the MLWA model and plotted as dashed-line curve in the same figure. This correction leads to redshift and broadening of the plasmon peak. Despite differences in modeling such as particle shapes, coupling with neighboring particles, and presence of an inhomogeneous medium, the modified quasistatic dipolar approximation is in good agreement with the FDTD model, which is a more realistic method. These results show that the MLWA method is as good as the FDTD method to account for the optical properties of the present structures.

Owing to the relative simplicity of the MLWA method, we can go one step further by including a correction for the natural oxide layer. The calculated spectrum of the particles coated with a 3-nm-thick aluminum oxide layer is plotted as dotted-line curve in Fig. 4. The inclusion of the natural oxide layer, while keeping the total thickness constant, leads to a redshift in the peak position of about $5 \mathrm{~nm}$. The redshifting due to the oxide layer is largely compensated by the shrink-

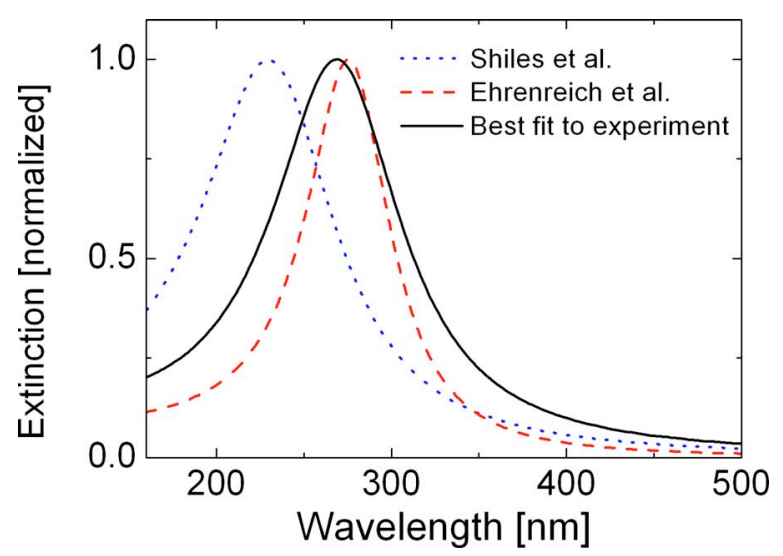

FIG. 5. (Color online) Calculated extinction spectra of Al nanoparticles of $40 \mathrm{~nm}$ total diameter and $30 \mathrm{~nm}$ total thickness with a $3 \mathrm{~nm}$ natural oxide layer using various optical constants. MWLA calculations are based on the optical constants reported in Refs. 7 and 33 (dotted line) and Ref. 34 (dashed line). The solid line curve represents the best fit to the experimental data presented in Fig. 3.

ing size of the metallic core, showing that the existence of the natural oxide layer has a small effect on the peak position. Ruling out these effects as possible sources of discrepancy between experiment and theory in Fig. 3, the only remaining factor is a possible difference between optical constants in experiment and theory.

In order to investigate the effect of the optical constants, we made further calculations based on various dielectric constants reported in literature, and the resulting spectra are plotted in Fig. 5. The optical constants used are extracted from optical measurements carried out by Shiles et al. ${ }^{33}$ and Ehrenreich et al. ${ }^{34}$ on clean and unoxidized thin films deposited and kept in UHV conditions. The results of the experiments by Shiles et al. ${ }^{33}$ were also adopted by the widely used reference book edited by Palik. ${ }^{7}$ Note that the dotted-line curves in Figs. 4 and 5 are identical in which both MLWA approximation and oxide-layer correction are incorporated. As seen in Fig. 5, the spectra based on different values of dielectric constants show significant variation. Within the range of wavelengths of 160-600 nm we obtained best-fit Drude parameters with a plasmon energy of $\hbar \omega_{p}=15.6 \mathrm{eV}$ and an electron relaxation time of $\tau=1.1$ fs for the values of Shiles et al., ${ }^{33}$ while the best-fit parameters are reported as $12.7 \mathrm{eV}$ and $5.12 \mathrm{fs}$ in the optical frequencies by Ehrenreich et $a l .{ }^{34}$ In fact, a survey of reported optical constants in the literature reveals an even greater variation than the values selected here. ${ }^{7,35}$ Since the dipolar model with the MLWA approximation is a simple analytical model, we carried out a best-fit procedure to the position and width of the experimental peak shown in Fig. 3 by changing the Drude parameters. The Drude parameters obtained with this procedure are $\hbar \omega_{p}=13.1 \mathrm{eV}$ and $\tau=1.0$ fs. These are reasonable values and within the range of experimentally reported data in literature. It should be noted that the obtained Drude parameters are simply fit parameters and this fitting procedure, which makes many assumptions, cannot be used for accurate determination of the optical properties of the real material. Nevertheless, the results demonstrate that the optical constants reported in literature show significant variations and 


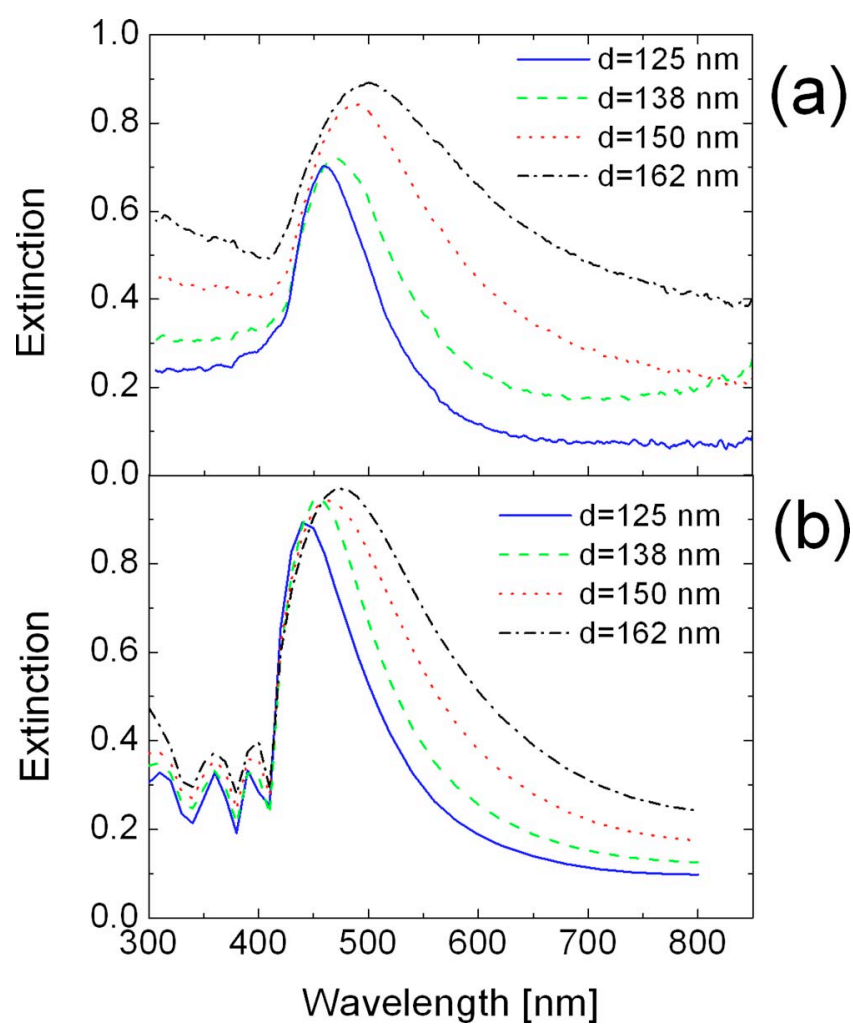

FIG. 6. (Color online) (a) Measured and (b) calculated extinction spectra of $\mathrm{Al}$ particle arrays with various diameters as provided in the figure. The array period is $280 \mathrm{~nm}$ and the metal thickness is $30 \mathrm{~nm}$. For the FDTD calculations the optical constants listed in Ref. 7 were used.

that the actual optical constants of a structure are subject to change depending on fabrication procedure. In particular, bulk oxidation during the deposition and electron scattering by the grain boundaries substantially change the optical properties of physically deposited Al. These properties are highly sensitive to the deposition rate and the base pressure of the deposition chamber. ${ }^{36}$ In conclusion, the optical constants of deposited Al films can vary and the discrepancy of $40 \mathrm{~nm}$ in peak position between theory (FDTD) and experiment can be attributed to the changes in optical constants. It should be noted that size effects, i.e., reduced relaxation times due to small particle size, become important at diameters below $20 \mathrm{~nm}$ and are negligible in the present case. However, the relaxation time can be reduced due to grain boundaries, defects, and roughness.

Figure 6 shows the measured and calculated extinction spectra of Al nanoparticles with various diameters. The metal thickness and the period of the array are fixed as 30 and 280 $\mathrm{nm}$, respectively, so that the evolution of the plasmon resonance peak with changing particle diameter can be demonstrated. With increasing diameter the peak position shifts to longer wavelengths and the peaks get broader due to increased retardation effects. The discrepancy in the peak positions between experiment and theory due to the optical constants is about 20-25 nm. Although other optical constants, as in Fig. 5, would have provided somewhat better agreement of the calculations with the experiment, we used the optical constants reported in Ref. 7 since they are generally accepted values. The calculated spectra show some

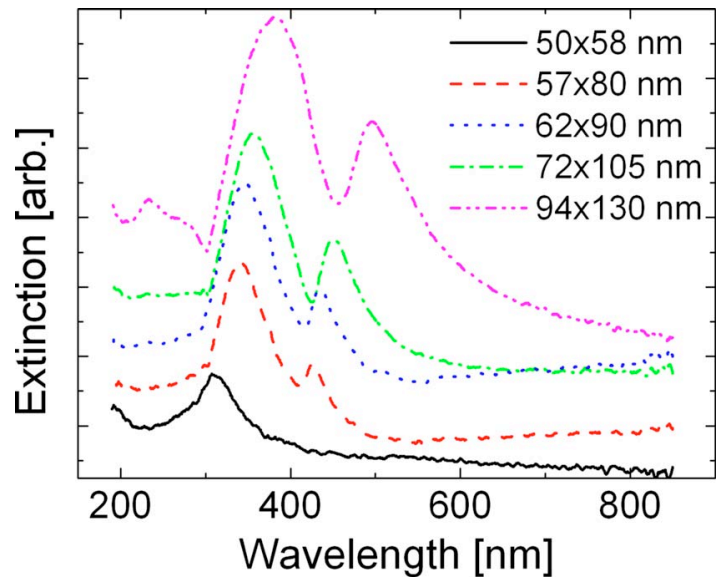

FIG. 7. (Color online) Measured extinction spectra of ellipsoidal Al nanoparticles of various sizes and aspect ratios. The perpendicular axis with respect to the substrate (film thickness) is $30 \mathrm{~nm}$ and the primary axes on the surface plane are provided in the figure. The period of the structures is 200 nm.

sharp modulations below $400 \mathrm{~nm}$ wavelength, which are not seen in the experiment. Both experiment and theory are zeroth-order transmission spectra, and the first-order diffraction channel is open at a wavelength of $410 \mathrm{~nm}$. Sharp attenuations in the intensities around the critical angles of the diffraction channels are known as Wood's anomalies, ${ }^{37}$ which are probably smeared out in the experiment due to inhomogeneous broadening.

Figure 7, finally, shows the extinction spectra of ellipsoidal nanoparticles with various sizes and aspect ratios. For the smallest particle, which has an aspect ratio close to unity, only one peak is visible. With increasing particle size and aspect ratio, two distinct peaks appear, which can be attributed to the plasmon resonances of the principle axes of the ellipsoids on the surface plane. The plasmon resonance associated with the short axis shifts from 310 to $380 \mathrm{~nm}$ with increasing length, whereas the long axis resonance shifts from 425 to $495 \mathrm{~nm}$. Since there are equal numbers of nanorods along two orthogonal directions, as seen in Fig. 2(c), the two different modes cannot be excited independently by using polarized light. This type of multiple resonance associated with different plasmon resonances of the different axes of asymmetric particles provides great flexibility in tunability of the plasmon resonances over a large wavelength range and is also advantageous in some applications, ${ }^{4,38}$ for example, for the "lightning rod" effect in which near fields at the ends of nanorods are substantially higher than those of spherical nanoparticles. Whereas Au and Ag nanorods exhibit plasmon resonances in the NIR range, $\mathrm{Al}$ nanorods can be easily tuned to the wavelengths in the visible range. Al nanorods can thus be used for plasmon-enhanced spectroscopy experiments in this range instead of $\mathrm{Au}$ and $\mathrm{Ag}$ spherical nanoparticles.

\section{CONCLUSIONS}

In summary, we have demonstrated the feasibility of achieving strong and sharp plasmon resonances in the UV region in $\mathrm{Al}$ nanoparticles and nanorods. Lithographic fabrication of nanoparticles with uniform size and shape properties was the key to avoiding severe broadening and redshift 
that obscure such resonances in random ensembles. The retardation effects are relatively significant for Al nanoparticles and therefore quasistatic dipolar approximation fails to describe the measured spectra, whereas the MLWA (Refs. 31 and 32) is found to be successful. Analysis of the experimental results points to small but significant variation in the optical constants compared with previously reported values. We developed a fitting procedure to extract the effective optical constants of metallic nanoparticles from their plasmon resonances.

Most importantly, the observation of sharp plasmon resonances of $\mathrm{Al}$ nanoparticles shows the feasibility of extending plasmonics research down to the deep-UV region, which has remained relatively unexplored. Thus, new applications such as surface-enhanced Raman spectroscopy (SERS) in this spectral region may be conceivable. In fact, UV-SERS experiments have been performed by using transition metals that exhibit only moderate enhancement factors but are nevertheless better than $\mathrm{Au}$ and $\mathrm{Ag} .{ }^{39}$ In contrast, Raman scattering enhancement of Al nanoparticles was theoretically predicted to be as high as $10^{5}$ and below $400 \mathrm{~nm}$. Al outperforms all metals except $\mathrm{Li}$ in terms of near-field enhancement $^{40}(\mathrm{Li}$, however, is not a suitable material for practical applications due to its high chemical activity).

Other interesting applications of $\mathrm{Al}$ plasmonic resonance might be in energetic materials and propellants. Great advances have, for example, been made in the development of nanoenergetic materials and $\mathrm{Al}$ nanoparticles have attracted significant attention as a promising candidate. ${ }^{41}$ Thus, in investigating fundamental processes such as oxidation and rupture of Al nanoparticles, monitoring their plasmonic resonances may be used as a simple or even in situ method complementing SEM and TEM analyses.

\section{ACKNOWLEDGMENTS}

This work was supported by the ETH Zurich Initiative on Composite Doped Metamaterials (CDM) and by ETH Research Grant (Grant No. TH-29/07-3). Part of this work was performed at the Swiss Light Source (SLS), Paul Scherrer Institute, Switzerland.

${ }^{1}$ C. F. Bohren and D. R. Huffman, Absorption and Scattering of Light by Small Particles (Wiley, New York, 1983).

${ }^{2} \mathrm{U}$. Kreibig and M. Vollmer, in Optical Properties of Metal Clusters, Springer Series in Material Science Vol. 25 (Springer, Berlin, 1995).

${ }^{3}$ G. C. Schatz and R. P. Duyne, in Handbook of Vibrational Spectroscopy, edited by J. M. Chalmers and P. R. Griffiths (Wiley, Chichester, 2002).

${ }^{4}$ E. Hutter and J. H. Fendler, Adv. Mater. (Weinheim, Ger.) 16, 1685 (2004).

${ }^{5}$ Y. Xia and N. J. Halas, MRS Bull. 30, 338 (2005).
${ }^{6}$ P. B. Johnson and R. W. Christy, Phys. Rev. B 6, 4370 (1972).

${ }^{7}$ D. Y. Smith, in Handbook of Optical Constants of Solids, edited by E. D. Palik (Academic, Orlando, FL, 1985).

${ }^{8}$ J. J. Wang, F. Walters, X. M. Liu, P. Sciortino, and X. G. Deng, Appl. Phys. Lett. 90, 061104 (2007).

${ }^{9}$ Y. Ekinci, H. H. Solak, C. David, and H. Sigg, Opt. Express 14, 2323 (2006).

${ }^{10}$ K. Ray, M. H. Chowdhury, and J. R. Lakowicz, Anal. Chem. 79, 6480 (2007).

${ }^{11}$ C. G. Granqvist, R. A. Buhrman, J. Wynsn, and A. J. Sievers, Phys. Rev. Lett. 37, 625 (1976).

${ }^{12}$ Y. H. Kim and D. B. Tanner, Phys. Rev. B 39, 3585 (1989).

${ }^{13}$ D. M. Wood and N. W. Ashcroft, Phys. Rev. B 25, 6255 (1982).

${ }^{14}$ R. Rojas, F. Claro, and R. Fuchs, Phys. Rev. B 37, 6799 (1988).

${ }^{15}$ C. G. Granqvist and O. Hunderi, J. Appl. Phys. 51, 1751 (1980).

${ }^{16}$ G. A. Niklasson, D. E. Aspnes, and H. G. Craighead, Phys. Rev. B 33, 5363 (1986).

${ }^{17}$ E. Anno and M. Tanimoto, Phys. Rev. B 64, 165407 (2001).

${ }^{18}$ G. L. Hornyak, K. L. N. Phani, D. L. Kunkel, V. P. Menon, and C. R. Martin, Nanostruct. Mater. 6, 839 (1995).

${ }^{19}$ M. J. Bloemer, M. C. Buncick, R. J. Warmack, and T. L. Ferrell, J. Opt. Soc. Am. B 12, 2552 (1988)

${ }^{20}$ J. Liu, A. I. Maaroof, L. Wieczorek, and M. B. Cortie, Adv. Mater. (Weinheim, Ger.) 17, 1276 (2005).

${ }^{21}$ J. Liu, B. Cankurtaran, G. McCredie, M. J. Ford, L. Wieczorek, and M. B. Cortie, Nanotechnology 16, 3023 (2005).

${ }^{22}$ P. E. Batson, Phys. Rev. Lett. 49, 936 (1982); Ultramicroscopy 9, 277 (1982).

${ }^{23}$ T. Stöckli, J.-M. Bonard, P.-A. Stadelman, and A. Chatelain, Z. Phys. D: At., Mol. Clusters 40, 425 (1997).

${ }^{24}$ Y. Ekinci, H. H. Solak, and C. David, Opt. Lett. 32, 172 (2007).

${ }^{25}$ H. H. Solak, Y. Ekinci, P. Käser, and S. Park, J. Vac. Sci. Technol. B 25, 91 (2007).

${ }^{26}$ Y. Ekinci, H. H. Solak, C. Padeste, J. Gobrecht, M. P. Stoykovich, and P. F. Nealey, Microelectron. Eng. 84, 700 (2007).

${ }^{27}$ A. Taflove and S. C. Hagness, Computational Electrodynamics: The Finite-Difference Time-Domain Method, 3rd ed. (Artech House, Boston, 2005).

${ }^{28}$ R. B. Pettit, J. Silcox, and R. Vincent, Phys. Rev. B 11, 3116 (1975).

${ }^{29}$ V. Y. Gertsman and Q. S. M. Kwok, Microsc. Microanal. 11, 410 (2005).

${ }^{30}$ C. Dahmen, B. Schmidt, and G. von Plessen, Nano Lett. 7, 318 (2007).

${ }^{31}$ L. Gunnarsson, T. Rindzevicius, J. Prikulis, B. Kasemo, M. Kall, S. Zou, and G. Schatz, J. Phys. Chem. B 109, 1079 (2005).

${ }^{32}$ K. L. Kelly, E. Coronado, L. L. Zhao, and G. Schatz, J. Phys. Chem. B 107, 668 (2003).

${ }^{33}$ E. Shiles, T. Sasaki, M. Inokuti, and D. Y. Smith, Phys. Rev. B 22, 1612 (1980).

${ }^{34}$ H. Ehrenreich, H. R. Phillip, and B. Segall, Phys. Rev. 132, 1918 (1963).

${ }^{35}$ C. L. Foiles, in Optical Properties of Pure Metals and Binary Alloys, Landolt-Börnstein, New Series, Group III, Vol. 15B, edited by K.-H. Hellwege and J. L. Olsen (Springer, Berlin, 1985).

${ }^{36}$ M. D. Tillin and J. R. Sambles, Thin Solid Films 167, 73 (1988); A. Shinya, Y. Okuno, M. Fukui, and Y. Shintani, Surf. Sci. 149, 371 (1997).

${ }^{37}$ R. W. Wood, Phys. Rev. 48, 928 (1935).

${ }^{38}$ J. W. M. Chon, C. Bullen, P. Zijlstra, and M. Gu, Adv. Funct. Mater. 17, 875 (2007)

${ }^{39}$ X.F. Lin, B. Ren, Z.-L. Yang, G.-K. Liu, and Z.-Q. Tian, J. Raman Spectrosc. 36, 606 (2005)

${ }^{40}$ E. J. Zeman and G. C. Schatz, J. Phys. Chem. 91, 634 (1987).

${ }^{41}$ D. D. Dlott, Mater. Sci. Technol. 22, 463 (2006); A. Rai, D. Lee, K. Park, and M. R. Zachariah, J. Phys. Chem. B 108, 14793 (2004). 